\title{
Correction to: Nuclear Radiation Monitoring in the Heterogeneous Internet of Things Era
}

Akbar Abbasi D, Fatemeh Mirekhtiary, and Hesham M.H. Zakaly

Correction to:

Chapter 4 in: F. Al-Turjman (ed.), Real-Time Intelligence for Heterogeneous Networks, https://doi.org/10.1007/978-3-030-75614-7_4

This chapter was inadvertently published without updating the following error.

The name and affiliation information of the author "Hesham M.H. Zakaly" was erroneously published in Chapter 4. This has been updated in this corrected version.

The updated online version of this chapter can be found at https://doi.org/10.1007/978-3-030-75614-7_4 